\title{
Central Compact Objects in Kes 79 and RCW 103 as 'Hidden' Magnetars with Crustal Activity
}

\author{
S. B. Popov ${ }^{1,4}$, A. A. Kaurov ${ }^{2}$ and A. D. Kaminker ${ }^{3}$ \\ ${ }^{1}$ Sternberg Astronomical Institute, Lomonosov Moscow State University, Universitetskii pr. 13, 119991 Moscow, Russia \\ ${ }^{2}$ Department of Astronomy and Astrophysics, The University of Chicago, Chicago, IL 60637, USA \\ ${ }^{3}$ Ioffe Physical-Technical Institute, Politekhnicheskaya 26, Saint Petersburg 194021, Russia \\ Email: polar@sai.msu.ru
}

(ReCEIVEd January 30, 2015; ACCEPTEd April 13, 2015)

\begin{abstract}
We propose that observations of 'hidden' magnetars in central compact objects can be used to probe crustal activity of neutron stars with large internal magnetic fields. Estimates based on calculations by Perna \& Pons, Pons \& Rea and Kaminker et al. suggest that central compact objects, which are proposed to be 'hidden' magnetars, must demonstrate flux variations on the time scale of months-years. However, the most prominent candidate for the 'hidden' magnetars — CXO J1852.6+0040 in Kes 79 - shows constant (within error bars) flux. This can be interpreted by lower variable crustal activity than in typical magnetars. Alternatively, CXO J1852.6+0040 can be in a high state of variable activity during the whole period of observations. Then we consider the source 1E161348-5055 in RCW103 as another candidate. Employing a simple 2D-modelling we argue that properties of the source can be explained by the crustal activity of the magnetar type. Thus, this object may be supplemented for the three known candidates for the 'hidden' magnetars among central compact objects discussed in literature.
\end{abstract}

Keywords: stars:individual (Kes 79, RCW 103) - stars:magnetars - stars:neutron.

\section{INTRODUCTION}

Magnetars - neutron stars (NSs) whose activity is related to dissipation of the magnetic field energy — have many different observational appearances (see recent reviews Mereghetti 2008; Rea \& Esposito 2011). Strong bursts observed in hard $\mathrm{X}$-rays are the most spectacular manifestations of this activity. What triggers magnetar bursts and long term outbursts ${ }^{1}$ is not known. Two main and probably causally associated approaches are discussed (see for example, Levin \& Lyutikov 2012 and references therein). Bursts are either related to crust cracks of some kind, or occur due to (possibly ensuing) magnetospheric activity (e.g., Parfrey, Beloborodov, \& Hui 2013; Beloborodov \& Levin 2014; Link 2014).

Magnetars activity is not uniform in time. There are periods of high state of activity (outbursts), and quiescent periods. The rate of energy release on long time scale is most likely driven by crustal processes, including field evolution in the crust. In order to compare relative importance and interplay of magnetospheric and crustal processes, it would be

\footnotetext{
${ }^{1}$ We use the term burst for a single event, and outburst for a long-term emission enhancement during which a few or many bursts can be observed.
}

useful to observe magnetars without crust activity (or with completely stable crust), but with evidences of active magnetospheric events, and magnetars with definitely suppressed magnetospheres, but with signs of active crustal processes. Then we would be able to conclude what triggers different types of activity: processes in the magnetosphere and/or in the crust.

Objects of the first kind are extremely difficult to identify, even though bare strange stars potentially can exist without a crust, (see e.g., Page \& Usov 2002 and references therein). As for the second kind of objects, three central compact objects (CCOs, see a review by de Luca 2008) are observed, which are believed to be so-called 'hidden' magnetars, for example like CXO J1852.6+0040 in Kes 79. The hypothesis of their suppressed magnetosphere is mainly based on the analysis of their thermal emission: pulse profiles of the X-ray light curves and a high pulse fraction, which requires magnetarscale fields in the crust (e.g., Shabaltas \& Lai 2012; Viganò $\&$ Pons 2012; Perna et al. 2013; Bogdanov 2014).

The idea of 'hidden' magnetars dates back to 1999, when the term was first proposed by Geppert, Page, \& Zannias (1999). Strong fall-back after a supernova explosion (Chevalier 1989) can lead to formation of an envelope which screens 
the magnetic field. Therefore, for an external observer the NS is visible as a source with low dipole field $B \sim 10^{10} \mathrm{G}$. Calculations show that an envelope with a mass $\sim 10^{-4} \mathrm{M}_{\odot}$ is enough to screen the field (see for example, Bernal, Page, $\&$ Lee 2013). However, these sources might be different from low-field magnetars (see Rea et al. 2014 and references therein), for which strong external multipoles are detected (Tiengo et al. 2013).

One can expect that observational features of 'hidden' magnetars are the following: (i) non-uniform surface temperature distribution, for instance, relatively small hot spots on the surface of an NS, (ii) large pulse fractions, (iii) flux variations on a time scale of months-to-years, which is typical for magnetars. In reality, candidates for the 'hidden' magnetars can manifest only some of these features. Anyway, 'hidden' magnetars can be important to probe initial properties of highly magnetized NSs (and, probably, the origin of huge magnetic field). For instance, their spin periods stay almost constant for several kyrs due to low spin-down rate (Popov 2013), and therefore initial rotation rate is 'frozen' in these objects. In the following sections, we present simple estimates which demonstrate that 'hidden' magnetars can be also used to probe properties of large magnetic fields in the NS crust.

\section{ANALYSIS AND ESTIMATES}

In this section, we produce simple estimates of crustal activity for different CCOs, which potentially can be 'hidden' magnetars, and present numerical model for the case of the CCO in RCW 103.

\subsection{Expected rate of $\mathrm{CCO}$ activity}

We start with estimates based on results by Perna \& Pons (2011) and Pons \& Rea (2012). These authors provide calculations of the rate and power of energy release events (ERE) in the crust of NSs, and then estimate their surface luminosity.

Since we consider sources similar to CXO J1852.6+0040 in Kes 79 (hereafter just Kes 79), typical ages of interest are about few kyrs. In terms of Perna \& Pons (2011), these sources are between 'young' and 'middle age' NSs. Depending on the parameter which characterises the fatigue limit of material subjected by magnetic stresses, these authors predict that for objects of this age EREs happen approximately once in a few years, with bimodal distribution of typical released energies $E_{\text {tot }} \sim 10^{40}-10^{41}$ ergs and $E_{\text {tot }} \sim 10^{43}-10^{44}$ ergs. Relative fraction of these two ERE types can change by a factor 2-3 in favour of one or another.

According to Pons \& Rea (2012), an ERE with $E_{\text {tot }} \sim$ few $\times 10^{43}$ ergs provides a surface luminosity above $2 \times 10^{34} \mathrm{erg} \mathrm{s}^{-1}$ for $\sim 100 \mathrm{~d}$ (their Figure 1). We expect that more than $10 \%$ of time the source is in a high state with enhanced luminosity ( $\approx$ once in three years; see Figure 2 of Perna \& Pons 2011). While in the high state, the pulse profile and pulsed fraction of $\mathrm{X}$-ray radiation may change, because

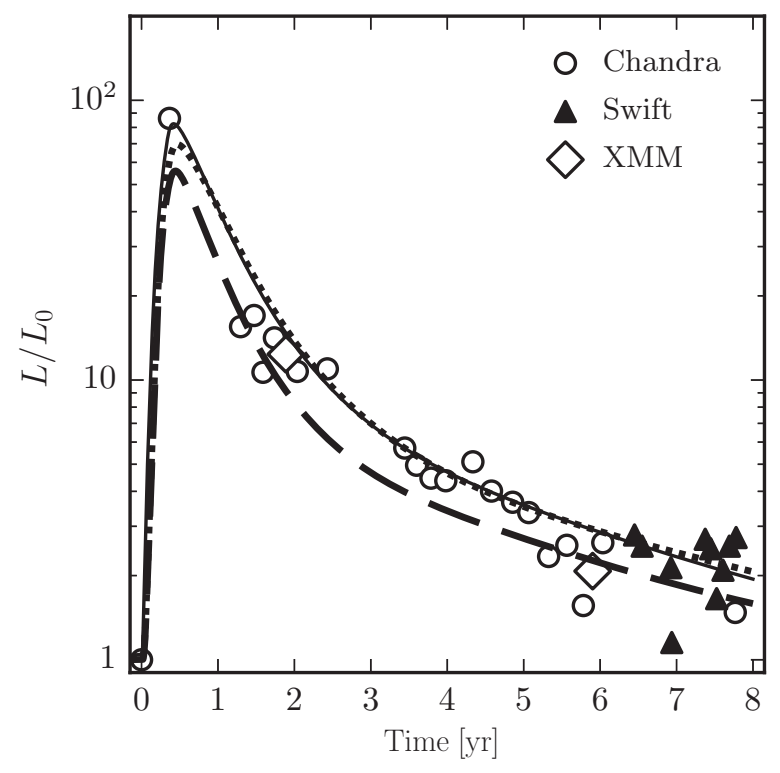

Figure 1. Simulated thermal X-ray luminosities $L$ as a function of time $t$ versus observational X-ray data on 1E 161348-5055 in the time interval from 1999 September to 2007 July (De Luca et al. 2008). Time zero corresponds to the first observation of the outburst. Observational fluxes and calculated luminosities are normalized to the flux $F_{0}$ and luminosity $L_{0}$ at the zero moment (see text). Three light curves correspond to three different heaters (see Table 1) in the NS crust located in layers $\rho_{1} \leq \rho \leq \rho_{2}$ within solid angles $\Delta \Omega_{0}$ around a symmetry axis (see text). Long dashed curve corresponds to the model (a) in Table 1; dotted and solid lines - to models (b) and (c), respectively. All heaters start energy release at the same moment and continue for $120 \mathrm{~d}$. The age of the NS at this moment is $t_{0}=2 \mathrm{kyr}$. Chandra data is shown with empty circles, XMM-Newton data - with diamonds, and Swift data — with filled triangles (error bars are disregarded).

the hot spots on the surface can form and move in respect to their relatively constant position in quiet state. The pulse profile then might become more complicated, and the pulsed fraction can increase, as well as decrease compared to the quiet state. The X-ray spectra in such states can be fitted by a sum of two blackbodies with temperatures corresponding to hot spots or the hot spot and the rest surface without activity.

Now we make estimates based on calculations by Kaminker et al. (2014, hereafter Paper I). These authors numerically modelled surface and neutrino luminosity of an NS as a result of a rather long (tens of kyrs) ERE in the crust. The key parameter is the heat rate $H\left[\mathrm{erg} \mathrm{cm}^{-3} \mathrm{~s}^{-1}\right]$. Paper I shows that the surface luminosity nearly saturates for $H \gtrsim 10^{20} \mathrm{erg} \mathrm{cm}^{-3} \mathrm{~s}^{-1}$. Here, we estimate $E_{\text {tot }}$ of an ERE with this heat rate.

First, we need to estimate the total volume in which energy is released. In Paper I, the authors used the thickness of the layer where energy is released $\sim 100 \mathrm{~m}$. Pons \& Rea (2012) used the layer with thickness $\sim 200 \mathrm{~m}$. The surface area of the region is typically given in terms of its angular size. In Paper I, the authors used the value $\sim 10^{\circ}$, and Perna \& Pons (2011) obtained $\sim 0.3-0.8$ radians for the emitting region. Altogether it gives a volume of $\gtrsim 10^{15} \mathrm{~cm}^{3}$. Duration of an ERE in Perna 
\& Pons (2011) is about one week (this is determined by the numerical resolution of the code), i.e $\sim 10^{6} \mathrm{~s}$. Then, we obtain that $E_{\text {tot }} \gtrsim 10^{41}$ ergs corresponds to $H \sim 10^{20} \mathrm{erg} \mathrm{cm}^{-3} \mathrm{~s}^{-1}$, (i.e., the regime of the most efficient heating). Thus, we can safely assume that most of the bursts in the NS of interest correspond to this regime with luminosity near the saturation level. Note that according to Perna \& Pons (2011) typical ERE has a total energy release $\gtrsim 10^{41}$ ergs.

For the chosen volume and duration of the ERE, the saturated luminosity is $L_{\text {surf }} \sim 10^{32.5} \mathrm{erg} \mathrm{s}^{-1}$, and characteristic neutrino emission is $L_{v} \sim 10^{35} \mathrm{erg} \mathrm{s}^{-1}$. Luminosity can be higher if larger volume and injected energy are involved. Indeed, according to Bogdanov (2014) the area of the hot anisotropic polar cap on the surface of the NS in Kes 79 is 5-10 times larger than the area of a hot spot with $10^{\circ}$ angular radius. So, it is necessary to use larger (by the same factor) energy release - for the same $H$, - than in the estimates above.

Let us apply these estimates to Kes 79 . The persistent Xray luminosity of Kes 79 is $\sim(4-5) \times 10^{33} \mathrm{erg} \mathrm{s}^{-1}$ (Viganò et al. 2013). Spin period is $P=0.105 \mathrm{~s}$, the pulsed fraction is rather high $(f \approx 60 \%)$, and the magnetic field is estimated from the period derivative as $B \sim 3 \times 10^{10} \mathrm{G}$ (see Viganò \& Pons 2012; Bogdanov 2014 and references therein).

Using the volume about an order of magnitude larger, i.e. $\gtrsim 10^{16} \mathrm{~cm}^{3}$, and so a larger characteristic heating power $\gtrsim 10^{36} \mathrm{erg} \mathrm{s}^{-1}$ (most of this energy is emitted by neutrinos), we obtain the surface (photon) luminosity $L_{\text {surf }} \gtrsim$ $10^{33.5} \mathrm{erg} \mathrm{s}^{-1}$, compatible with the luminosity of Kes 79 . It corresponds to the persistent total energy losses (approximately equal to neutrino losses) $E_{\text {tot }} \lesssim 10^{42}$ ergs per week. On this background, $\mathrm{X}$-ray radiation of any powerful outbursts could be discernible. If the source produces EREs with $E_{\text {tot }} \sim 10^{43} \mathrm{erg}$, then the NS would be able to stay brighter than in a quiescent state for several months $(\sim 100 \mathrm{~d})$. As in the estimates above one could anticipate that $\sim 10 \%$ (or even more) of time the NS would be in the state of enhanced luminosity (and quite probably with modified pulse profile).

On the other hand, the set of data presented by Gotthelf, Halpern, \& Alford (2013) and Bogdanov (2014) with observations of Kes 79 every several months during several years shows that no significant variability has been detected. Thus, we can safely conclude that no significant variations in the crustal activity happened in this source during several years of observations. Therefore, we can treat this source as remaining in the quiescent state.

Alternatively, Kes 79 could be in active state during all the time of observations. So, additional energy releases simply were not visible at all, or visible only for a short periods of time (see Pons \& Rea 2012), and so were not detected on the background of relatively strong persistent crustal energy release.

The lack of observable bursting activity makes Kes 79 similar to the anomalous X-ray pulsar (AXP) CXOU J010043.1-721134 (Tiengo, Esposito, \& Mereghetti 2008). Actually, there are several AXPs which do not demonstrate bursts and have relatively stable X-ray flux, but CXOU J010043.1-721134 is the most inactive among them in terms of variations of the surface emission. Note, however, that this source was not monitored extensively, and some periods of higher or lower luminosity could be missed.

Thus, we can make an intermediate conclusion that observations of typical CCOs with very stable parameters, and in the first place - Kes 79, indicate that their crustal activity is different from that of majority of magnetars (contrary to the expectation (iii) in the Introduction), unless they are in active state during all time of observations. One can conclude that just the absence of large external magnetic field might be the reason for this.

However, one can expect that there is a chance of observing outbursts and other types of magnetar activity on the background of a quiescent X-ray radiation from other central sources. Such an example is given below.

\subsection{The case of 1E161348-5055 in RCW 103}

1E161348-5055 (hereafter 1E) is the central compact Xray source in the supernova remnant RCW103. The age of the remnant is about $2 \mathrm{kyr}$, and the central source has several peculiar properties. Several hypothesis about the nature of $1 \mathrm{E}$ have been proposed (see for example, Pizzolato et al. 2008 and references therein). Here, we suggest that this source can be treated as a 'hidden' magnetar with strong activity in the crust.

$1 \mathrm{E}$ is characterised by variable $\mathrm{X}$-ray emission in the range of luminosities $\sim 10^{33}-10^{35} \mathrm{erg} \mathrm{s}^{-1}$. This variability is long term (months-years) and irregular. In addition, a period of $6.67 \mathrm{~h}$ was found (De Luca et al. 2006). The nature of this period is not known and many hypotheses have been discussed in the literature, including compact binaries of different kind, etc. In any case, since the period seems to be very stable, the main possibility is that this is a spin period of an NS with the upper limit for the period derivative as $|\dot{P}|<1.6 \times 10^{-9} \mathrm{~s} \mathrm{~s}^{-1}$ (Esposito et al. 2011).

The spectra obtained in certain phases of activity can be fitted with two blackbodies with temperatures $\sim 0.5$ and $1 \mathrm{keV}$. At the same time pulse profile changes significantly in different phases. It was noted (De Luca et al. 2006) that pulsed fraction is lower and pulse shape is more irregular when the source is in a high state. All these features (except the period) naturally fit the picture of a 'hidden' magnetar with strong crustal activity (see De Luca et al. 2006, where the authors indicate similarity between properties of $1 \mathrm{E}$ and magnetars). Indeed, crustal activity can result in appearance of heated regions of the surface: lower temperature could correspond to normal cooling of an NS of a given age - typically $\lesssim 100-200 \mathrm{eV}$, and the higher one - to typical magnetar temperatures - about $0.5 \mathrm{keV}$. Therefore, in the simplest case the spectrum could be fitted as a sum of two blackbodies, and the pulse profile would be modified, correspondingly. Note, that in the case of $1 \mathrm{E}$ we face a more complicated situation with two different bright regions at the stellar surface (see 
above). Anyway, time scale of X-ray variability and typical luminosity of $1 \mathrm{E}$ are in correspondence with the range of time intervals between neighbour EREs for magnetars of similar age (Perna \& Pons 2011).

$1 \mathrm{E}$ is significantly younger than Kes 79 ( $\sim 2 \mathrm{kyr} v s .6 \mathrm{kyr}$ ), and so bursts are expected to happen more often. Small period derivative $^{2}$ would be also consistent with expectations for 'hidden' magnetars.

\subsection{Outbursts and relaxation in 1E161348-5055}

Figure 1 shows results of simplified simulations employing 2D-code (see Paper I) of luminosity-time dependencies, $L(t)$, imposed on the X-ray data on long outburst of $1 \mathrm{E}$ in the time interval from 1999 September to 2007 July. The X-ray fluxes $F(t)$ measured in different moments by different missions (all presented in De Luca et al. 2008) have been normalised to the first observation in this series, when the object is presumably in a low state $\left(F / F_{0}=L / L_{0}\right)$. The calculated curves are normalised to the luminosity $L_{0}=1.37 \times 10^{33} \mathrm{erg} \mathrm{s}^{-1}$ corresponding to a standard cooling of an NS (without heating) with $M=1.4 \mathrm{M}_{\odot}$ at the age $2 \mathrm{kyr}$. We assume that the observational outbursts are powered by the energy of the magnetar's magnetic fields hidden in the bulk (deep in the crust) of the star. The magnetic energy is supposed to transform in the heat inside an internal region(s) of the NS crust. We investigate qualitatively possible parameters of the magnetar heater which are needed to provide observable EREs with $\sim$ two orders enhancement of the observable flux and about ten years of relaxation.

As shown in Paper I, the results of calculations of thermal radiation from NSs with internal heaters just weakly depend on the employed equation of state (EOS) of the nucleon matter in the stellar core. Therefore, we perform the illustrative calculations with the use of a toy-model EOS, following Paper I. In the core, we use the simple parametrisation suggested by Heiselberg \& Hjorth-Jensen (1999) for the EOS obtained by Akmal, Pandharipande, \& Ravenhall (1998). The maximum mass of NSs in this toy-model is $2.16 \mathrm{M}_{\odot}$, and powerful direct Urca process in the core is allowed as $M \geq 1.77 \mathrm{M}_{\odot}$. We use the model for NS mass $1.4 \mathrm{M}_{\odot}$. The corresponding circumferential stellar radius is $R=12.74 \mathrm{~km}$, and the central density $\rho_{\mathrm{c}}=7.78 \times 10^{14} \mathrm{~g} \mathrm{~cm}^{-3}$. Such a star without internal heaters would cool rather slowly (standard cooling, e.g., Yakovlev et al. 2001) via modified Urca process of neutrino emission from the core. For simplicity, we neglect effects of General Relativity in our 2D-calculations including a redshift of the surface luminosity.

As usual in cooling calculations, the star is divided into the bulk interior and a thin outer heat-blanketing envelope (e.g., Gudmundsson, Pethick, \& Epstein 1983) which extends from the surface to the layer of the density $\rho=\rho_{\mathrm{b}} \sim 10^{10} \mathrm{~g} \mathrm{~cm}^{-3}$. Its thickness is about $200 \mathrm{~m}$. In the bulk interior $\left(\rho>\rho_{\mathrm{b}}\right)$, the $2 \mathrm{D}$ code solves the full set of thermal evolution equations

\footnotetext{
${ }^{2}$ If it was confirmed with better precision
}

to simulate the cooling of NSs with the internal axially symmetric heater in the crust. The neutrino emissivities, $Q_{v}$, are taken from Yakovlev et al. (2001). In the present version, we neglect effects of magnetic fields on thermal conduction and neutrino emission, as well as on properties of the blanketing envelope. In this envelope, the updated version of the code (see Potekhin, Chabrier, \& Yakovlev 2007, for details) uses a solution of stationary one-dimensional equations for hydrostatic equilibrium and thermal structure with radial heat transport.

Similar to Paper I, we introduce an internal phenomenological heat source located in a layer at $\rho_{1} \leq \rho \leq \rho_{2}$. The basic difference is only in the scale of temporal behaviour of the heater. The heating rate $H\left[\mathrm{erg} \mathrm{cm}^{-3} \mathrm{~s}^{-1}\right]$ is taken in the form

$$
H=H(\rho, t)=H_{0} \Theta(\rho, \theta, t),
$$

where $H_{0}$ is the characteristic heat intensity and $\Theta(\rho, \theta, t)$ is the step function, which equals unity when $\left(\rho_{1}<\rho<\rho_{2}\right)$ and $\left(\theta<\theta_{0}\right) \&\left(t_{0}<t<t_{0}+\tau\right)$, and vanishes outside these spatial and temporal regions. The heater looks like a hot wide axisymmetric slab limited by the angle $\theta_{0}$ and by densities $\rho_{1}$ and $\rho_{2}$ along radial axis. The solid angular size of the heater can be expressed as $\Delta \Omega_{0} / 4 \pi=\left(1-\cos \theta_{0}\right) / 2$. The temporal parameters are fixed in our model: the moment of the energy-input onset, $t_{0}$, is $2 \mathrm{kyr}$, the duration of energy input, $\tau$, is chosen to be $120 \mathrm{~d}$ in order to match the interval between the first two observations.

In our model, there are five main free parameters: $H_{0}, \rho_{1}$, $\rho_{2}, \Delta \Omega_{0} / 4 \pi$ (or $\theta_{0}$ ), and $\tau$.

We adopt three set of parameters (labelled '(a)-(c)') which are listed in Table 1. The choice of parameters is not motivated by any formal fitting and we do not attempt to perfectly match the observations. We only try to reproduce the general shape of the light curve.

There is a variety of possibilities for the composition and the amount of the accretion matter capable to screen completely the magnetar's magnetic field discussed in the literature (e.g., Houck \& Chevalier 1991; Viganò \& Pons 2012; Bernal et al. 2013). In order to account for it, we choose the models (a) and (b) to be identical except the composition of the blanketing envelopes. Two possible compositions are considered: the ground state and accreted matter (labelled as 'iron' and 'accr'. in Table 1, correspondingly). The former composition is the ground state matter: iron is the main constituent up to $\rho=10^{8} \mathrm{~g} \mathrm{~cm}^{-3}$, heavier elements dominate at higher density (e.g., Haensel, Potekhin, \& Yakovlev 2007). The latter one corresponds to a fully accreted envelope (see Potekhin et al. 2003) composed successively of H, He, C, O with boundaries dependent on $\rho$ and $T$ between the layers. In deeper layers, composition of an accreted crust transits to iron.

In contrast to Paper I, we use a relatively short time of the energy input, $\tau=120 \mathrm{~d}$, to simulate long outbursts of $1 \mathrm{E}$. Therefore, the main features of the present calculations are 
Table 1. Parameters of three heaters used for simulations represented in Figure 1 for the $1.4 \mathrm{M}_{\odot}$ star and heating time $\tau=120 \mathrm{~d}$.

\begin{tabular}{lccccc}
\hline \hline Model & $H_{0}\left(\mathrm{erg} \mathrm{cm}^{-3} \mathrm{~s}^{-1}\right)$ & $\rho_{1}\left(\mathrm{~g} \mathrm{~cm}^{-3}\right)$ & $\rho_{2}\left(\mathrm{~g} \mathrm{~cm}^{-3}\right)$ & $\Delta \Omega_{0} / 4 \pi$ & Composition \\
\hline (a) & $3.0 \times 10^{20}$ & $10^{11}$ & $10^{12}$ & 0.4 & iron \\
(b) & $3.0 \times 10^{20}$ & $10^{11}$ & $10^{12}$ & 0.4 & accr. \\
(c) & $3.0 \times 10^{19}$ & $4 \times 10^{10}$ & $4 \times 10^{11}$ & 0.6 & accr. \\
\hline \hline
\end{tabular}

the following: a rapid increase of luminosity and 10-yr long relaxation tail shown in Figure 1.

Full energy input in the three considered cases is $E_{\text {tot }} \sim$ $10^{44} \mathrm{erg}$, and efficiency of thermal radiation from the surface is $\sim 0.01$ as the released energy mostly goes to the neutrino emission. This energy input allows one to achieve the maximum luminosity about $10^{35} \mathrm{erg} \mathrm{s}^{-1}$ and thereby provides two orders increase of the luminosity.

Similar results are obtained for blackbody temperature $T_{\mathrm{s}}(t)$ of the outburst. The maximum temperature, $\sim$ $(3.5-4.0) \times 10^{6} \mathrm{~K}$, is followed by a long (>8 yr) decay. However, the temperature calculated in such a way characterises only an averaged value over a considerable part of the stellar surface (see Table 1). Moreover, observational data on temperature are more scarce than data on fluxes. And we prefer to use the latter one to confront calculations and observations.

Let us emphasize that parameters of a heater are introduced purely phenomenologically. They allow one to outline the behaviour of the outburst luminosities with time just approximately. Nevertheless, our results allow to place constraints on possible models and parameters, including the total heat energy, the size of the hot region in the stellar crust, and the ratio of densities $\rho_{2} / \rho_{1}$ which regulates the tail endurance.

A theoretical model of the internal heating of the 'hidden' magnetars is far out of the scope of this paper. In application to the standard magnetars, it was noticed (e.g. by Kaminker et al. 2012) that the required heat intensity $H_{0} \sim 10^{20} \mathrm{erg}$ $\mathrm{cm}^{-3} \mathrm{~s}^{-1}$ could be consistent with Ohmic decay of electric currents within the heater. However, it is still not clear how to transport the magnetic energy stored in the bulk of the star to the localised heater inside the crust and what is the structure of magnetic fields in the heater surroundings. These problems concern both types of magnetars that we discuss. Presumably, some progress in describing these processes has been made recently (e.g. Beloborodov \& Levin 2014).

\section{DISCUSSION AND CONCLUSIONS}

\subsection{Spin period of $1 \mathrm{E} 161348-5055$}

Interpretation and explanation of the period of $1 \mathrm{E}$ is problematic in any scenario. Let us make some simple estimates to check if it is in principle possible to bring the value $P \sim 6.67$ $\mathrm{h}$ in correspondence with the 'hidden' magnetar scenario. Note, if we assume that $1 \mathrm{E}$ is a 'hidden' magnetar, then it is difficult to spin down the NS significantly during its lifetime, and we are left with processes close to the moment of its birth.

Usually, three distinct phases of an NS evolution are defined: ejector, propeller, and accretor (see for example, Lipunov 1992). Ejector spin-down is not effective enough to reach long periods in a few hours (typical time before the fall-back onset) even in the case of very large magnetic fields. Something stronger is required. When the fall-back is already initiated (which happens on the time scale $\sim 10^{4} \mathrm{~s}$ ), but before the quasi-stable supercritical accretion is settled (e.g., Bernal, Lee, \& Page 2010; Bernal et al. 2013), it is possible that a short propeller stage is present (Shvartsman 1970; Illarionov \& Sunyaev 1975). Spin evolution at the propeller stage can be, in the first approximation, described as (Lipunov 1992)

$$
\frac{\mathrm{d}(I \omega)}{\mathrm{d} t}=-k \frac{\mu^{2}}{R_{\mathrm{A}}^{3}},
$$

where $I$ is moment of inertia of an NS, $\omega$ is its spin frequency, $\mu$ is magnetic moment, and $R_{\mathrm{A}}$ is Alfven (magnetospheric) radius. The coefficient $k$ can be frequency dependent, but for rough estimates it can be taken as a constant of order unity. Then, a characteristic time scale of complete spin-down is

$$
\tau \sim I \omega_{0} R_{\mathrm{A}}^{3} \mu^{-2},
$$

where $\omega_{0}$ is the initial spin frequency. For initial spin period about few milliseconds and magnetar-scale field, we have an approximate relation:

$$
\tau \sim k_{\mathrm{mag}}\left(R_{\mathrm{A}} / R_{\mathrm{NS}}\right)^{3} \mathrm{~s} .
$$

Here, $R_{\mathrm{NS}}$ is the NS radius, and all dependencies on the initial spin period and the field are included in the coefficient $k_{\text {mag }}$, which is $\lesssim 1$ for typical magnetar and fall-back parameters. At the stage of fall-back, the Alfven radius can be very small, close to the NS surface. Thus, it is possible to reduce significantly rotation of an NS during a very short time period. Then, after the stage of supercritical accretion rotation can be nearly frozen down. Anyway, the considerations above can be acceptable only as qualitative estimates.

Moreover, an NS with large magnetic field can spin-down before the onset of the stage of fall-back, i.e. before the moment when the reverse shock makes its way back to the NS surface. Such a situation is possible if the magnetosphere of a newborn NS interacts with the expanding envelope in 
the propeller-like regime. However, detailed study of this case is beyond the scope of this paper.

Alternatively, $1 \mathrm{E}$ can reach long spin period if there is a fossil disc around this compact object, and its external magnetic field is large as for a typical magnetar, i.e. if the object is not a 'hidden' magnetar. Interaction of highly magnetised NS with a disc might result in slow rotation, so that the period $P=6.67 \mathrm{~h}$ can be reached in $2 \mathrm{kyr}$ (see De Luca et al. 2006; Pizzolato et al. 2008 and references therein). However, in this case one has to explain why we do not observe any manifestation of a strong magnetospheric activity in this source (flares, non-thermal radiation, etc.).

Although, we mentioned an example (see above) of a magnetar without significant transient activity - AXP CXOU J010043.1-721134. So, potentially, we need longer observation of RCW 103 to be sure that there is no large external magnetic field of this compact object.

\subsection{Unification of NSs}

The idea of unifying different types of young isolated NSs in one evolutionary framework (Kaspi 2010) looks very promising (see for example, Viganò et al. 2013; Igoshev, Popov, \& Turolla 2014 and references and discussions therein). The concept of buried magnetic field (Bernal et al. 2010; Ho 2011; Viganò \& Pons 2012) might be once more ingredient necessary to fulfill the program of 'Grand unification of neutron stars'. In particular, it can help to link CCOs with other types of young NSs including magnetars. However, Bogdanov, Ng, \& Kaspi 2014 presented arguments against the hypothesis that after few to tens of thousand years CCOs could become normal radio pulsars when their fields diffuse out.

To establish possible evolutionary links between different types of young NSs, it is necessary to better understand all types of activity they demonstrate. In this respect, it would be useful to address in more details the problem of crustal activity in CCOs proposed to be 'hidden' magnetars.

Of course, it is not expected that all of CCOs are 'hidden' magnetars. Long-term behaviour of the majority of CCOs demonstrates that magnetic field evolution in their crusts is different from that in magnetars. For most of CCOs, it is consistent with a notion that their buried fields have values typical to normal radio pulsars. Indeed, most of them do not show any significant activity, or even variability. Pulsed fraction is low in most of the sources. However, it is worth to mention that Krause et al. (2005) discovered specific features in the supernova remnant Cas A which can be interpreted as light echo of the past activity of a magnetar (see a brief discussion in de Luca 2008).

There is a significant probability that the source 1E161348-5055 in RCW103 (or 1E) is a 'hidden' magnetar, as its properties can be well explained by the crustal activity of the magnetar type. Note, that its properties are in contrast with the puzzling absence of variability of CXO J1852.6+0040 (or Kes 79), which was also proposed to be a 'hidden' magnetar. If we assume that Kes 79 is in a low (quiescent) state, then it is natural to argue that crusts of 'hidden' magnetars are not as active as crusts of normal magnetars. The activity of $1 \mathrm{E}$ is also relatively moderate: only three outbursts in $\sim 20 \mathrm{yr}$ of observations were detected - less than expected for a magnetar of such age.

On the other hand, differences between Kes 79 and 1E could be related to the amount of fall-back. For larger accreted masses, the initial crust can be shifted down significantly deeper, and so the energy release can happen in the region of larger density, that hinders the energy transport to the stellar surface (energy is carried away mainly by neutrinos). Finally, different levels of activity can be attributed to different initial toroidal magnetic fields. This possibility was discussed by Perna \& Pons (2011) and Pons \& Perna (2011).

Another CCO - RX J0822-4300 in Puppis A - also demonstrates some peculiarities (see de Luca et al. 2012 and references therein). This source has two antipodal hot spots with different temperatures and sizes. In addition, a variable spectral line has been detected. Potentially, these features can be related to field structure in the crust, and so the object can be linked with the population of 'hidden' magnetars.

If some of CCOs are indeed 'hidden' magnetars, then we can consider an interesting possibility. Presumably, the amount of fall-back inversely depends on the energy of explosion (MacFadyen, Woosley, \& Heger 2001; Perna et al. 2014). In a recent paper, Chugai \& Utrobin (2014) demonstrated that the energy of an explosion grows with stellar mass: $E \sim M^{3.8}$ (however, it is not clear, if the same scaling can be used for stars in close interacting binaries, and if magnetars are related to SN IIP). With this result in hands, we can assume that standard and 'hidden' magnetars have progenitors with different masses: higher masses for standard magnetars without fall-back, and so with larger energies of explosion. If a crustal magnetic field can reach large values for standard as well as for 'hidden' magnetars, then (despite several opposite claims that magnetars are related to the most massive NS progenitors, e.g. Muno et al. 2006), it is quite likely that mass is not the crucial factor determining strength of the magnetic field. Then, it is quite reasonable to consider effects of the initial rotation rate of NSs.

Isolated stars (or stars in wide binaries) cannot produce rapidly rotating cores (see a recent paper by Maeder \& Meynet 2014 and reference therein) which are necessary for generation of magnetar magnetic fields. Therefore, the idea of magnetar origin in close binaries (Popov \& Prokhorov 2006; Bogomazov \& Popov 2009), which is supported by observation (Davies et al. 2009; Clark et al. 2014) obtains an additional support.

In this respect, it is tempting to note, that the remnant of SN 1987A potentially can be a 'hidden' magnetar, as it was probably born soon after a coalescence (Morris \& Podsiadlowski 2007), and so rotation of the stellar core could be significantly enhanced, which is favourable for magnetar field generation. Strong fall-back advocated in the case of SN 
1987A (Chevalier 1989; Houck \& Chevalier 1991; Bernal et al. 2010) indirectly supports this hypothesis.

\section{ACKNOWLEDGMENTS}

SP thanks Yuri Levin and Konstantin Postnov for discussions. The authors thank Alexander Potekhin for data of the accreted (heatblanketing) envelope calculations. SP was supported by the RFBR grant 14-02-00657. ADK was supported partly by the RFBR grant 14-02-00868 and by the State Program 'Leading Scientific Schools of RF' (grant NSh 294.2014.2). We thank the anonymous referee for useful comments.

\section{REFERENCES}

Akmal, A., Pandharipande, V. R., \& Ravenhall, D. G. 1998, PhRvC, 58,1804

Beloborodov, A. M., \& Levin, Y. 2014, ApJL, 794, L24

Bernal, C. G., Lee, W. H., \& Page, D. 2010, RMxAA, 46, 309

Bernal, C. G., Page, D., \& Lee, W. H. 2013, ApJ, 770, 106

Bogdanov, S. 2014, ApJ, 790, 94

Bogdanov, S., Ng, C.-Y., \& Kaspi, V. M. 2014, ApJL, 792, L36

Bogomazov, A. I., \& Popov, S. B. 2009, ARep, 53, 325

Chevalier, R. A. 1989, ApJ, 346, 847

Chugai, N. N., \& Utrobin, V. P. 2014, AstL, 40, 291

Clark, J. S., Ritchie, B. W., Najarro, F., Langer, N., \& Negueruela, I. 2014, A\&A, 565, A90

Davies, B., Figer, D. F., Kudritzki, R.-P., Trombley, C., Kouveliotou, C., \& Wachter, S. 2009, ApJ, 707, 844

De Luca, A. 2008, in AIP Conf. Proc., Vol. 983, 40 Years of Pulsars: Millisecond Pulsars, Magnetars and More, ed. C. Bassa, Z. Wang, A. Cumming, \& V. M. Kaspi (Melville, New York), 311

De Luca, A., Caraveo, P. A., Mereghetti, S., Tiengo, A., \& Bignami, G. F. 2006, Science, 313, 814

De Luca, A., Mignani, R. P., Zaggia, S., Beccari, G., Mereghetti, S., Caraveo, P. A., \& Bignami, G. F. 2008, ApJ, 682, 1185

de Luca, A., et al. 2012, MNRAS, 421, L72

Esposito, P., Turolla, R., de Luca, A., Israel, G. L., Possenti, A., \& Burrows, D. N. 2011, MNRAS, 418, 170

Geppert, U., Page, D., \& Zannias, T. 1999, A\&A, 345, 847

Gotthelf, E. V., Halpern, J. P., \& Alford, J. 2013, ApJ, 765, 58

Gudmundsson, E. H., Pethick, C. J., \& Epstein, R. I. 1983, ApJ, 272, 286

Haensel, P., Potekhin, A. Y., \& Yakovlev, D. G., eds. 2007, Astrophysics and Space Science Library, Vol. 326, Neutron Stars 1: Equation of State and Structure (New York: Springer), 115

Heiselberg, H., \& Hjorth-Jensen, M. 1999, ApJL, 525, L45

Ho, W. C. G. 2011, MNRAS, 414, 2567

Houck, J. C., \& Chevalier, R. A. 1991, ApJ, 376, 234
Igoshev, A. P., Popov, S. B., \& Turolla, R. 2014, AN, 335, 262

Illarionov, A. F., \& Sunyaev, R. A. 1975, A\&A, 39, 185

Kaminker, A. D., Kaurov, A. A., Potekhin, A. Y., \& Yakovlev, D. G. 2012, in ASP Conf. Ser., Vol. 466, Electromagnetic Radiation from Pulsars and Magnetars, ed. W. Lewandowski, O. Maron, \& J. Kijak (San Francisco: Astronomical Society of the Pacific), 237

Kaminker, A. D., Kaurov, A. A., Potekhin, A. Y., \& Yakovlev, D. G. 2014, MNRAS, 442, 3484

Kaspi, V. M. 2010, PNAS, 107, 7147

Krause, O., et al. 2005, Science, 308, 1604

Levin, Y., \& Lyutikov, M. 2012, MNRAS, 427, 1574

Link, B. 2014, MNRAS, 441, 2676

Lipunov, V. M. 1992, Astrophysics of Neutron Stars (Berlin: Springer-Verlag)

MacFadyen, A. I., Woosley, S. E., \& Heger, A. 2001, ApJ, 550, 410

Maeder, A., \& Meynet, G. 2014, ApJ, 793, 123

Mereghetti, S. 2008, A\&ARv, 15, 225

Morris, T., \& Podsiadlowski, P. 2007, Science, 315, 1103

Muno, M. P., et al. 2006, ApJL, 636, L41

Page, D., \& Usov, V. V. 2002, PhRvL, 89, 131101

Parfrey, K., Beloborodov, A. M., \& Hui, L. 2013, ApJ, 774, 92

Perna, R., Duffell, P., Cantiello, M., \& MacFadyen, A. I. 2014, ApJ, 781, 119

Perna, R., \& Pons, J. A. 2011, ApJL, 727, L51

Perna, R., Viganò, D., Pons, J. A., \& Rea, N. 2013, MNRAS, 434, 2362

Pizzolato, F., Colpi, M., De Luca, A., Mereghetti, S., \& Tiengo, A. 2008, ApJ, 681, 530

Pons, J. A., \& Perna, R. 2011, ApJ, 741, 123

Pons, J. A., \& Rea, N. 2012, ApJL, 750, L6

Popov, S. B. 2013, PASA, 30, 45

Popov, S. B., \& Prokhorov, M. E. 2006, MNRAS, 367, 732

Potekhin, A. Y., Chabrier, G., \& Yakovlev, D. G. 2007, Ap\&SS, 308, 353

Potekhin, A. Y., Yakovlev, D. G., Chabrier, G., \& Gnedin, O. Y. 2003, ApJ, 594, 404

Rea, N., \& Esposito, P. 2011, in High-Energy Emission from Pulsars and their Systems, ed. D. F. Torres, \& N. Rea (Berlin Heidelberg: Springer-Verlag), 247

Rea, N., Viganò, D., Israel, G. L., Pons, J. A., \& Torres, D. F. 2014, ApJL, 781, L17

Shabaltas, N., \& Lai, D. 2012, ApJ, 748, 148

Shvartsman, V. F. 1970, R\&QE, 13, 1428

Tiengo, A., Esposito, P., \& Mereghetti, S. 2008, ApJL, 680, L133

Tiengo, A., et al. 2013, Nature, 500, 312

Viganò, D., \& Pons, J. A. 2012, MNRAS, 425, 2487

Viganò, D., Rea, N., Pons, J. A., Perna, R., Aguilera, D. N., \& Miralles, J. A. 2013, MNRAS, 434, 123

Yakovlev, D. G., Kaminker, A. D., Gnedin, O. Y., \& Haensel, P. 2001, PhR, 354, 1 